\title{
PLASMA BNP LEVEL AS PREDICTOR TOOL OF MYOCARDIAL INFARCTION IN DIABETIC PATIENTS
}

\author{
SHIMPA SHARMA ${ }^{1}$, SHARMA R.C. ${ }^{2}$, SHARMA R.K. ${ }^{3}$ AND DESALE H. ${ }^{1}$ \\ 1Department of General Medicine, Padmashree Dr. D.Y. Patil Medical College, Quatre Bornes Mauritius. \\ 2Department of Physiology, Faculty of Medicine, School of Biomedical Sciences, AIMST University, Malaysia. \\ ${ }^{3}$ Department of Obstetrics and Gynecology, Padmashree Dr. D.Y. Patil Medical College, Quatre Bornes Mauritius. \\ *Corresponding Author: Email- drsharmax@gmail.com
}

Received: February 26, 2012; Accepted: April 09, 2012

\begin{abstract}
Early detection of asymptomatic left ventricular systolic dysfunction (LVSD) or diastolic dysfunction (DD) in Type II Diabetes Mellitus patients may prevent complication such as Myocardial infarction. Present study is aimed to study the prediction chances of myocardial infarction in type II Diabetes mellitus patients using co-relation of plasma BNP level with 2-D-ECHO findings. A total of 100 diabetes mellitus type II patients were studied of which $50 \mathrm{DM}$ had evidence of $\mathrm{Ml}$ and remaining 50 were without any evidence of Ml for more than $5 \mathrm{yrs}$. BNP values were inversely proportional to left ventricular systolic ejection fraction. This correlation was significant $(p<0.001)$. Elevated BNP levels were significantly correlated with presence of Diastolic Dysfunction $(p<0.001) . I n$ our study the BNP cutoff value of $100 \mathrm{pg} / \mathrm{ml}$ had a sensitivity of $90 \%$, specificity of $70 \%$. In patients with only Diabetes, of the 14 patients with elevated BNP levels and normal Ejection Fraction, 10 revealed diastolic dysfunction. Of the remaining 4, further follow-up of two patients showed presence of Coronary Artery Disease, indicates that BNP is good investigation for detecting underlying coronary artery disease in high risk population such as Type II Diabetes Mellitus.
\end{abstract}

Citation: Shimpa Sharma, et al (2012) Plasma BNP level as predictor tool of Myocardial infarction in Diabetic Patients. International Journal of Medical and Clinical Research, ISSN:0976-5530 \& E-ISSN:0976-5549, Volume 3, Issue 4, pp.-143-146.

Copyright: Copyright@2012 Shimpa Sharma, et al. This is an open-access article distributed under the terms of the Creative Commons Attribution License, which permits unrestricted use, distribution, and reproduction in any medium, provided the original author and source are credited.

\section{Introduction}

Discovery of the cardiac peptides in the 1980s renewed the appreciation of the heart as an endocrine organ. It also opened a new area of cardiovascular-renal physiology, raising hope for further diagnostic and therapeutic progress in the management of heart failure and condition causing heart failure [1]. . B-type natriuretic peptide (BNP) is a 32-amino-acid polypeptide secreted by the cardiac ventricles in response to ventricular volume expansion and pressure overload [2, 3, and 4]. It is released in a wide range of cardiovascular disease, including systolic and diastolic left ventricular dysfunction, coronary artery disease, valvular heart disease, acute and chronic right ventricular failure, and left and right ventricular hypertrophy secondary to arterial or pulmonary hypertension. Serum Brain Natriuretic Peptide (BNP) assessment has demonstrated utility for prognostic stratification of patients with heart failure and coronary artery disease (CAD). increased BNP levels in CAD patients have higher rate of cardiac complications and higher mortality post MI. Observational studies have suggested that, when used in conjunction with other clinical information, B -type natriuretic peptide levels may be useful in establishing or ruling out the diagnosis of heart failure $[5,6,7,8,9]$. However, it has been reported that variety of endocrine, paracrine, and autocrine factors that are initiated in heart failure, including norepinephrine, angiotensin II, glucocorticoids, and proinflammatory cytokines known to stimulate the BNP expression and release[10]. Yamamoto et al proved conclusively that BNP is superior to both $\mathrm{N}$ terminal and C-terminal ANP as a marker for ventricular systolic or diastolic dysfunction and ventricular hypertrophy in patients with, or at risk for, cardiac disease [11]. Specifically, BNP demonstrated greater sensitivity and specificity than $\mathrm{N}$-terminal or Cterminal ANP for reductions in ejection fraction or increases in left ventricular (LV) mass [12]. Cowie et al. has evaluated levels of natriuretic peptides in assessment of patients with possible new CHF and observed BNP had the highest sensitivity (97\%) and specificity $(84 \%)$ for the diagnosis of CHF in the primary care outpatient setting [13].

The Present study has been undertaken to measure plasma BNP levels in patients of MI with DM as well as diabetic patients with- 
out history of MI or IHD to evaluate the cardiac status using relationship of BNP Level with 2D-ECHO. It is also attempted to predict the chances of myocardial infarction in the high risk population group of patients with Type II Diabetes Mellitus.

\begin{abstract}
Materials \& Methods
A total of 100 patients comprise of 50 cases of Type II DM with MI and remaining 50 cases are of only Type II DM without history of Ml were studied prospectively over a period of 2 years from October 2008 through October 2010. The cases selected amongst the general population in and around hospital and were subjected to detail history, clinical examination and routine laboratory investigations for diabetic profile.

Left ventricular ejection fraction (LVEF) was estimated by visual assessment of LV contractile performance and wall motion in multiple 2-dimensional views. The LVEF was categorized as normal (>=60\%), moderately diminished (44\%-59\%), and severely impaired (30\%-44\%). The accuracy of such visual assessment of LVEF has been validated previously [14]. Diastolic function was estimated by spectral Doppler. Systolic and diastolic dysfunction was defined as ejection fraction $<50 \%$ with global hypokinesia or discrete wall motion abnormalities along with diastolic dysfunction as described.
\end{abstract}

\section{Measurement of BNP Plasma Levels}

Blood sample for BNP estimation was collected in plastic tubes using EDTA plasma is used for the AxSYM BNP assay which is based on MEIA technology (Micro particle Enzyme Immuno Assay). It is 2-step micro particle enzyme immunoassay using BNP specific monoclonal antibodies that has been developed for use on the automated AxSYM analyzer. The analyte is captured using anti-BNP coated microparticles with detection via anti-BNP conjugated alkaline phosphatase and measurement of fluorescence produced from the breakdown of a 4-methylumbelliferyl phosphate substrate. The assay has a dynamic range of $0-4,000 \mathrm{pg} / \mathrm{ml}[15$, 16].

\section{Statistical Analysis}

Statistical analysis of BNP values was carried out using "analyzeit" software for comparison of both the group using $t$ tests for independent samples and ANOVA. Results are expressed as mean \pm SE for raw values. Sensitivity, specificity, and accuracy were computed for BNP using a appropriate cut points. Prognostic utility of the BNP level was computed using receiver operating curve (ROC) and expressed the results as area under the curve with $95 \% \mathrm{Cl}$ for this area.

\section{Observations and Results}

The analysis of observations of all the 100 cases of group $A$ and $B$ revealed that Males $(n=62)$ demonstrated that higher mean BNP values $(74.51 \pm 47$ and $824.14 \pm 47 \mathrm{pg} / \mathrm{ml}$ respectively $)$ than Females $(69.85 \pm 34$ and $727.54 \pm 47 \mathrm{pg} / \mathrm{ml}$ respectively $)$ irrespective of evidence of CAD. However the difference between BNP level of male and female was statistically insignificant and did not demonstrate relevance of BNP level with gender variation.

In group A, 49 patients were found to be with normal ejection fraction (above 60\%) except one patient who had ejection fraction 55 $\%$. Diastolic dysfunction was seen in 23 patients with normal ejec- tion fraction. In group B, 48 patients were found to be with abnormal ejection fraction ranging from 60 to $30 \%$ while 2 patients were with ejection fraction above $60 \%$. 34 patients of group had Diastolic dysfunction with poor ventricular ejection fraction below $50 \%$. Analysis of BNP level and its relevance with ejection fraction, diastolic function in diabetic patients is summarized in table -1. It is observed that mean BNP level $(72.18 \mathrm{pg} / \mathrm{ml})$ in diabetic patients without any evidence of $\mathrm{Ml}$ were significantly reduced as compared to mean BNP level $(799.02 \mathrm{pg} / \mathrm{ml})$ observed in DM patients associated with symptoms of Ml. It is seen that asymptomatic diabetic patients of

group $A$, who were found to have normal left ventricular function on echo but with presence of diastolic dysfunction patients have demonstrated lower BNP level $(150.04 \mathrm{pg} / \mathrm{ml})$ as compared to BNP Level $(682.54 \mathrm{pg} / \mathrm{ml})$ seen in symptomatic diabetic patients with evidence of $\mathrm{Ml}$ and difference was found to be statistically significant. Further statistically significant elevated BNP level $(910.25 \mathrm{pg} / \mathrm{ml})$ were seen in patients having DM with depressed ventricular and diastolic function as compared to asymptomatic patients, who were found to have abnormal left ventricular function on echo without MI. ROC analysis was carried out to assess the ability of plasma BNP level to determine its predictive value in patients with normal versus abnormal left ventricular function. It is seen that area under the ROC curve, using BNP to separate normal versus abnormal left ventricular function in patients with evidence of $\mathrm{Ml}$ was $0.86(95 \% \mathrm{Cl} 0.83-0.94, \mathrm{P}<0.001)$. A BNP cutoff value of $150 \mathrm{pg} / \mathrm{ml}$ had a sensitivity of $86 \%$, a specificity of $72 \%$, and an accuracy of $82 \%$. Levels of $\geq 120 \mathrm{pg} / \mathrm{ml}$ had a positive predictive value of $93 \%$. In group A, ROC analysis revealed that plasma BNP to separate normal versus abnormal left ventricular function was less than that for those of group $B$, but still highly significant (AUC $=0.81,95 \% \mathrm{Cl} 0.71-0.91, \mathrm{P}<0.001)$. In addition, a BNP cutoff value of $100 \mathrm{pg} / \mathrm{ml}$ had a sensitivity of $90 \%$, a specificity of $70 \%$, and an overall accuracy of $79 \%$.

\section{Discussion}

Asymptomatic left ventricular dysfunction demonstrated humoral activation characterized by increases in the natriuretic peptides without activation of the circulating renin-angiotensin system (18). In the present study, 21 of the 31 asymptomatic patients who had an abnormal echo as well as diastolic dysfunction, with BNP levels of $910.60 \mathrm{pg} / \mathrm{ml}$. While BNP levels cannot be used by itself to differentiate between systolic and diastolic dysfunction, a low BNP level in the setting of normal systolic function by echocardiography can likely rule out clinically significant diastolic dysfunction (11). Echocardiography is the gold standard in diagnosing LV dysfunction and helpful in identifying the presence and extent of dysfunction. However, limited availability, cost considerations, and subjective variations attribute of expertise performing the examination \& evaluating the report has been a constraint for use of this technique. Further 2-D-ECHO is used more as diagnostic tool hence predictive value of this tool is very poor in cases of LV Dysfunction.

In our study we found that cut off of $100 \mathrm{pg} / \mathrm{ml}$ had $90 \%$ sensitivity and $70 \%$ specificity. This correlates with one of the largest multinational prospective studies by Maisel et al in [18] reporting BNP cutoff value of $100 \mathrm{pg} / \mathrm{ml}$ had $90 \%$ sensitivity and $76 \%$ specificity for differentiating heart failure from other causes of dyspnea. In 
the study conducted by Dao et al, a BNP cutoff value of $80 \mathrm{pg} / \mathrm{ml}$ had $98 \%$ sensitivity and $92 \%$ specificity. In the study conducted by McCullough et al, a BNP cutoff value of $50 \mathrm{pg} / \mathrm{ml}$ had $94 \%$ sensitivity and $70 \%$ specificity.

BNP values and LVEF were shown to have an inversely proportional relationship in this study. This relationship was stronger in the group of patients who had Diabetes with Myocardial Infarction. Of the 48 patients with LV Dysfunction, 44 patients had BNP level more than $100 \mathrm{pg} / \mathrm{ml}$. In cases of patients with Diabetes Mellitus without history or evidence of Ischemic Heart Disease, $(N=50)$ only one patient had LV dysfunction. Even this patient had BNP of more than $100 \mathrm{pg} / \mathrm{ml}$. The statistical significance of this correlation between BNP and LVEF in this study was $p=0.005$. In our study, the mean BNP values of patients with Normal ejection fraction i.e $>=60 \%$ is $77.35 \mathrm{pg} / \mathrm{ml}$. In patients with $<60 \%$ EF the mean BNP level is $911.19 \mathrm{pg} / \mathrm{ml}$. Three other investigations have attempted to establish the relationship between plasma BNP and Cardiac function in patients referred for echocardiography. [17, 18, 19]. In the first study by Yamamoto et al. , in which the end point of Ejection fraction of $45 \%$ was found in $11 \%$ of the cohort the negative predictive value ("rule out" function) of BNP was high enough $(96 \%)$ to make BNP a useful discriminator. In the second study by Maisel et al.[18], which used a broader end point of ejection fraction of $50 \%$ or Doppler evidence of diastolic dysfunction, the end point prevalence was $47 \%$ in the cohort and was associated with good sensitivity (86\%) and excellent specificity (98\%). The third study Lubien et al. attempted to detect diastolic dysfunction only, which was found in $40 \%$ of the patient cohort and BNP showed good specificity (98\%) and positive predictive value (96\%). In summary, studies of patient cohorts referred for echocardiography show that high BNP concentration is associated with abnormal Cardiac function. It is apparent that BNP has good negative predictive ("rule out") value. Lubien and colleagues[19] measured plasma BNP levels in 294 patients referred for echocardiography and found that patients with abnormal LV diastolic function ( $N$ $=119$ ) had a mean plasma BNP concentration of $286 \pm 31 \mathrm{pg} / \mathrm{ml}$ while the normal LV group ( $N=175)$ had a mean BNP concentration of $33 \pm 3 \mathrm{pg} / \mathrm{ml}$. Plasma concentrations were particularly elevated in patients with restrictive filling patterns and in those with symptoms. In the study by Lubien et. al, a BNP value of $62 \mathrm{pg} / \mathrm{ml}$ gave a sensitivity of $85 \%$ and specificity of $83 \%$ for detecting isolated diastolic dysfunction.

In our study, the plasma BNP value of $100 \mathrm{pg} / \mathrm{ml}$ gave a sensitivity of $77.2 \%$ and a specificity of $62.8 \%$ for detecting diastolic dysfunction. In this study, therefore of 60 patients with elevated BNP levels, there were 45 patients with Left ventricular systolic dysfunction (LVSD) and 44 with diastolic dysfunction. Only 5 patients showed no 2D-ECHO evidence of cardiac dysfunction. In view of the correlation of elevated BNP levels with cardiac dysfunction, we attempted to further investigate those patients with high BNP and normal 2-D Echo study. This is outside the purview of this study. However, we were able to motivate 2 of the 5 patients for further investigations. One showed positive Treadmill test, while the other had a positive coronary Angiographic study. This finding has encouraged us to consider a further study of correlating BNP levels with findings on Treadmill test. While 2-D ECHO identifies existing dysfunction and damage. The predictive value of the Treadmill Test would make the considered study of immense preventive value. The relationship between the elevated $B N$ levels in patients with Diastolic dysfunction (DD) and the lower BNP levels in those without Diastolic dysfunction (DD) is noted in both the above studies. The comparatively higher levels of BNP in our study are probably due to the fact that we have not excluded patients with Left ventricular systolic dysfunction (LVSD).

\section{Conclusion}

In this study, elevated BNP levels showed good correlation with Cardiac Dysfunction - either systolic as measured by Ejection Fraction or Diastolic dysfunction as seen on 2-D-Echo with Spectral Color Doppler. BNP level with cut-off of 100 gave us a sensitivity of $90 \%$ \& specificity of $70 \%$ with $p$ value of $<0.001$. 2 Out of 5 Patients with elevated BNP levels but normal 2D-ECHO also showed presence of underlying $C A D$ on further investigations. Both patients thus diagnosed had Type II Diabetes Mellitus. This indicates that BNP is good investigation for detecting underlying coronary artery disease in high risk population such as Type II Diabetes Mellitus.

We conclude that BNP levels can be considered as a diagnostic tool in the detection of cardiac dysfunction and/or underlying coronary artery disease especially in high-risk groups like Type II Diabetes Mellitus. Further studies with higher patient numbers and correlation of BNP levels with treadmill test would help in providing more accurate data.

Table1

\begin{tabular}{|lllll|}
\hline S.N. Parameters & Group A & Group B & P Value \\
\hline 1 & Mean BNP Level & 72.18 & 799.02 & P>.001 \\
3 & Mean BNP Level in Male & 74.51 & 824.14 & - \\
\hline & $\begin{array}{l}\text { Mean BNP Levels in Female } \\
\text { Mean BNP Level in patients with normal }\end{array}$ & 69.85 & 727.54 & - \\
$\begin{array}{l}\text { Ventricular ejection fraction and diastolic 150.04 } \\
\text { dysfunction }\end{array}$ & 682.54 & $\mathrm{P}>.001$ \\
$\begin{array}{l}\text { Mean BNP Level in patients with sup- } \\
\text { pressed Ventricular ejection fraction and 482.54 } \\
\text { diastolic dysfunction }\end{array}$ & 910.25 & $\mathrm{P}>.001$ \\
\hline
\end{tabular}

\section{References}

[1] Braunwald E., Harrison D.C., Chidsey C.A. (1964) Am. J. Med., 36, 1-4.

[2] Dao Q., Krishnaswamy P., Kazanegra R. (2001) J. Am. Coll. Cardiol, 37, 379-85.

[3] Morrison L.K., Harrison A., Krishnaswamy P., Kazanegra R., Clopton P., Maisel A. (2002) J. Am. Coll. Cardiol, 39, 202-9.

[4] Cabanes L., Richaud-Thiriez B., Fulla Y. (2001) Chest, 120, 2047-50

[5] Yasue H., Yoshimura M., Sumida H. (1994) Circulation, 90, 195-203.

[6] Hama N., Itoh H., Shirakami G. (1995) Circulation, 92, 155864.

[7] Wiese S., Breyer T., Dragu A. (2000) Circulation, 102, 3074-9.

[8] Davis M., Espiner E., Richards G. (1994) Lancet, 343, 440-4.

[9] McDonagh T.A., Robb S.D., Murdoch D.R. (1998) Lancet, 351, 9-13.

[10]Iwanaga Y., Nishi I., Furuichi S. (2006) J. Am. Coll. Cardiol, 47, 742-8.

[11]Yamamoto K., Burnett J.C., Jougasaki M., Nishimura R.A., Bailey K.R., Saito Y., Nakao K., Redfield M.M. (1996) Hypertension, 28, 988-994. 
[12]Chen H.H., Burnett J.C. (1999) Assoc. Am. Physicians, 111, 406-416.

[13]Cowie M.R., Struthers A.D., Wood D.A., Coats A.J., Thompson S.G., Poole-Wilson P.A., Sutton G.C. (1997) Lancet, 350, 1349-53.

[14]Willenheimer R.B., Israelsson B.A., Cline C.M., Erhardt L.R. (1997) Scand Cardiovasc J., 31, 9-16.

[15]Shimizu H., Alono K., Masuta K., (1999) Clin Chim Acta, 285, $169-172$. 